\title{
Analysis of the Power of Discrimination between Normal Distribution and Weibull Distribution of the Goodness-of-Fit Tests
}

\author{
Ramona CLINCIU \\ Transilvania University of Brasov, Romania, r.clinciu@unitbv.ro
}

\begin{abstract}
The paper presents and emphasizes the capacity of different goodness-of-fit tests to discriminate between normal distribution and Weibull distribution, from the acceptance of the normality hypothesis point of view. The Weibull and the normal distribution are met frequently in the reliability analysis. The discrimination between these two distributions is difficult by considering the graphical representation of the data considered. The Weibull distribution is accepted as being a normal distribution by some goodness-of-fit tests. Since data analysis is very important in the evaluation of the reliability of a system, it results that it is very important to determine correctly the distribution which describes the data best. On this purpose, goodness of fit tests are to be conducted. The goodness of fit tests which were taken into account are both general goodness-of-fit tests Kolmogorov-Smirnov, Cramer-von-Mises, Anderson-Darling, and normality goodness-of-fit tests - Lilliefors, Shapiro-Wilk, D'Agostino, Massey, Filliben, Z, Cox. These goodness-of-fit tests are conducted on Weibull distributed data in order to test their power to discriminate between Weibull and normally distributed data.
\end{abstract}

\section{Keywords}

metrological reliability, goodness-of-fit tests, normal distribution, Weibull distribution

\section{Introduction}

It is known that data analysis performs a very important role in the quantitative evaluation of system reliability and maintainability. One of the most important issues in this data analysis is to find the best, or the most appropriate distribution to describe the collected data. In order to determine the distribution which fits the data best, one has to conduct goodness-of-fit tests.

The Weibull and the normal distribution are met very often in the reliability analysis. The discrimination between these two distributions is difficult to accomplish only by considering the graphical representation (histograms) of the data considered. This issue is due to the fact that, for certain values of the shape parameter $\beta$ of the Weibull distribution function $(3<\beta<4)$ [4], these distributions are very much alike. Also, the Weibull distribution is accepted as being a normal distribution by some goodness-of-fit tests.

The purpose of this research came from the necessity to test the normality of the data especially in metrology, for the analysis of the metrological reliability. General metrology uses especially the normal distribution, although positive and asymmetrical distributions, as for example Weibull distribution, are met frequently in the analysis of the metrological reliability. In these cases, it is necessary to perform goodness-of-fit tests in order to ascertain that the normal distribution fits the data best The aim of this research is to conduct goodness of fit tests on Weibull distributed data in order to determine the values of the shape parameter of the Weibull distribution function for which the analysed data is considered to obey a normal distribution function. Since the quality of the results of a data analysis depends on the goodness-of-fit test which was chosen to determine if the normal distribution fits the data best, different goodness of fit tests were taken into account.

This paper aims to present and emphasize the results obtained by conducting different goodnessof-fit tests, both general goodness-of-fit tests and normality goodness-of-fit tests, on Weibull distributed data, in order to test the power of discrimination the considered tests between Weibull and normally distributed data.

The effect of the size of the sample on the discrimination power of the goodness-of-fit tests which were considered is also presented and emphasized in the paper. 


\section{Generation of Weibull Distributed Data}

In order to test the capability of the goodness-of-fit tests for the discrimination between the Weibull and the normally distributed data, a series of experiments have been conducted. They are based on the generation of Weibull distributed data, using different values for the shape parameter $\beta$ of the Weibull distribution function.

The reliability function for the biparametrical Weibull distribution $(\gamma=0$, where $\gamma$ is the location parameter of the Weibull distribution) is given by $[2,4]$ :

$$
R(x)=\exp \left[-\left(\frac{x}{\eta}\right)^{\beta}\right],
$$

where $\eta$ is the scale parameter and $\beta$ is the shape parameter of the Weibull distribution function.

One can write also $[2,4]$ :

$$
R(x)=1-F(x),
$$

where $F(x)$ is the distribution function

From equations (1) and (2), it is possible to get the expression for the random variable $x_{i}$ which has a Weibull distribution:

$$
x_{i}=\exp \left[\frac{1}{\beta}\left(\ln \left(\ln \frac{1}{1-F_{n}\left(x_{i}\right)}\right)+\beta \ln \eta\right)\right],
$$

where $F_{n}\left(x_{i}\right)$ is the empirical distribution function and it is calculated from [4]:

$$
F_{n}\left(x_{i}\right)=\frac{i-0.3}{n+0.4} \text {. }
$$

\section{Study of the Power of Discrimination of the Goodness-of-fit Tests}

The goodness-of fit tests considered in this research are general goodness-of-fit tests - KolmogorovSmirnov, Cramer-von-Mises, Anderson-Darling, and normality goodness-of-fit tests - Lilliefors, Shapiro-Wilk, D'Agostino, Massey, Filliben, Z, Cox.

All the goodness-of-fit tests considered were applied according to the prescriptions found in the speciality literature [1-6].

The general goodness-of-fit tests were used in their modified form, taken into account the situation in which the parameters of the normal distribution are not known and they need to be estimated by $[1,2]$ :

$$
\begin{gathered}
m=\frac{1}{n} \sum_{i=1}^{n} x_{i}, \\
\sigma=\sqrt{\frac{1}{n-1} \sum_{i=1}^{n}\left(x_{i}-m\right)^{2}},
\end{gathered}
$$

where $x_{i}, i=1, \ldots n$, are the values in the sample, $n$ is the size of the sample, $m$ is the mean, and $\sigma$ is the standard deviation.

Although in [6] Cox goodness-of-fit test is considered to be proper for $n>100$, it was conducted on small sizes of samples too and the results are presented in this paper. Some of the results presented in this research can contribute to different statistical analyses as those presented in $[7,8]$.

The research has been realized by considering samples with different sizes $n \in\{5,10,20,30,40$, $50,60,80,100,120\}$. Each sample has been generated according to the equation (3). The values of the parameters of the Weibull distribution considered are: $\gamma=0, \eta=1$ and $\beta \in(1,10)$. 
RECENT, Vol. 21, no. 3(62), 2020

The goodness-of-fit tests which were considered were used to test the normality of the data, although they were conducted on Weibull distributed data, on samples with different sizes $n \in\{5,10$, $20,30,40,50,60,80,100,120\}$, each sample being generated according to the equation (3).

The reason to conduct goodness-of-fit tests on Weibull distributed data and to check the normality hypothesis is to determine the values of the shape parameter $\beta$ of the Weibull distribution function for which the considered goodness-of-fit tests ascertain that the normal distribution fits the data.

Table 1 presents the results obtained by considering samples with sizes $n \in\{5,10,20,30,40,50\}$, while Table 2 presents the results obtained by considering samples with sizes $n \in\{50,60,80,100$, $120\}$. The results indicate the bounds of the interval for the shape parameter $\beta$ of the Weibull distribution function. For all values within the intervals indicated, the normal distribution was accepted by the tests as representing the data.

Table 1. Values for the shape parameter $\beta$ of the Weibull distribution for which the normal distribution was accepted by the tests as representing the data, $n \in\{5,10,20,30,40\}$

\begin{tabular}{|c|c|c|c|c|c|}
\hline \multicolumn{7}{|c|}{ Sample size $(n)$} & 5 & 10 & 20 & 30 & 40 \\
\hline \multicolumn{7}{|c|}{ General goodness-of-fit tests } \\
\hline Kolmogorov - Smirnov & $1-10$ & $1-10$ & $1-10$ & $1-10$ & $1.25-10$ \\
\hline Cramer-von-Mises & $1-10$ & $1-10$ & $1.25-10$ & $1.25-10$ & $1.5-10$ \\
\hline Anderson-Darling & $1-10$ & $1-10$ & $1.25-10$ & $1.5-10$ & $1.5-10$ \\
\hline \multicolumn{7}{|c|}{ Normality goodness-of-fit tests } \\
\hline Lilliefors & $1-10$ & $1-10$ & $1-10$ & $1-10$ & $1-10$ \\
\hline Shapiro-Wilk (3<n<50) & $2-10$ & $2-10$ & $2.25-9.5$ & $2.5-7.25$ & $2.75-5.75$ \\
\hline D'Agostino $(\mathrm{n}>50)$ & - & - & - & - & - \\
\hline Massey $(10<\mathrm{n}<30)$ & - & $1.5-10$ & $1.5-10$ & $1.5-10$ & - \\
\hline Filliben & $1.73-10$ & $2-10$ & $2.2-9.75$ & $2.25-7.75$ & $2.5-6.75$ \\
\hline $\mathrm{Z}$ & $1-10$ & $1.25-10$ & $1.5-10$ & $1.75-10$ & $2-10$ \\
\hline Cox & $2.92-3.92$ & $3.1-3.78$ & $3.2-3.75$ & $3.25-3.75$ & $3.27-3.76$ \\
\hline
\end{tabular}

Table 2. Values for the shape parameter $\beta$ of the Weibull distribution for which the normal distribution was accepted by the tests as representing the data, $\mathrm{n} \in\{50,60,80,100,120\}$

\begin{tabular}{|c|c|c|c|c|c|}
\hline \multicolumn{7}{|c|}{ Sample size (n) } & 50 & 60 & 80 & 100 & 120 \\
\hline \multicolumn{7}{|c|}{ General goodness-of-fit tests } \\
\hline Kolmogorov - Smirnov & $1.25-10$ & $1.25-10$ & $1.5-10$ & $1.5-10$ & $1.5-10$ \\
\hline Cramer-von-Mises & $1.5-10$ & $1.75-10$ & $1.75-10$ & $2-10$ & $2-10$ \\
\hline Anderson-Darling & $1.75-10$ & $1.75-10$ & $1.75-10$ & $2-10$ & $2-10$ \\
\hline \multicolumn{7}{|c|}{ Normality goodness-of-fit tests } \\
\hline Lilliefors & $1.25-10$ & $1.25-10$ & $1.5-10$ & $1.5-10$ & $1.5-10$ \\
\hline Shapiro-Wilk (3<n<50) & $3.35-4.75$ & - & - & - & - \\
\hline D'Agostino (n>50) & $1.5-10$ & $1.5-10$ & $1.5-10$ & $1.75-10$ & $1.5-10$ \\
\hline Massey (10<n<30) & - & - & - & & \\
\hline Filliben & $2.5-6$ & $2.55-5.8$ & $2.75-5.5$ & $3-4.75$ & $3-4.5$ \\
\hline $\mathrm{Z}$ & $2.25-10$ & $2.25-8.5$ & $2.25-7$ & $2.45-6.2$ & $2.5-6$ \\
\hline Cox & $3.29-3.76$ & $3.3-3.76$ & $3.32-3.77$ & $3.33-3.77$ & $3.3-3.77$ \\
\hline
\end{tabular}

\section{Conclusions}

Different goodness-of-fit tests have different power to discriminate between the Weibull and the normal distribution and since they are very important for the evaluation of the system reliability, it is 
useful to know which test is more adequate to a certain case. By analysing the results indicated in Table 1 and Table 2, the conclusion is that all the considered goodness-of-fit tests accept the normal hypothesis for a certain interval of values of the shape parameter $\beta$ of the Weibull distribution.

A first conclusion drawn from the research is that the discrimination power of the general goodness-of-fit tests is smaller than the discrimination power of the normality goodness-of-fit tests. This conclusion can be reached by analysing the values obtained for the shape parameter $\beta$ of the Weibull distribution, values for which the normal distribution was accepted by the tests as representing the data.

The most conclusive tests are: the Cox goodness-of-fit test, followed by Filliben and Z goodness-offit tests, which are based on the correlation coefficient and Shapiro-Wilk goodness-of-fit test. These tests restrict the considered interval of the shape parameter of the Weibull distribution to a relative small interval. Therefore, their power to discriminate between the two distributions - the Weibull and the normal distribution - is greater than the power of discrimination of other normality tests Lilliefors, D'Agostino or Massey goodness-of-fit tests, or of the general goodness-of-fit tests considered - Kolmogorov-Smirnov, Cramer-von-Mises or Anderson-Darling.

Another conclusion drawn from this research is that the power to discriminate between the Weibull and the normal distribution increases with the size of the sample.

\section{References}

1. Montgomery D.C., Runger G.C. (2003): Applied Statistics and Probability for Engineers. John Wiley \& Sons, Inc., ISBN 0-471-20454-4, http://www.um.edu.ar/math/montgomery.pdf

2. Kececioglu D. (2002): Reliability Engineering Handbook. Prentice Hall, ISBN 978-1-932078-00-8

3. Zio E. (2007): An Introduction to the Basics of Reliability and Risk Analysis. World Scientific, New Jersey, ISBN13: 978-981-279-639-3

4. Martinescu I., Popescu I. (1995): Fiabilitate (Reliability). Gryphon, Brasov, Romania, ISBN 973-604-007-0 (in Romanian)

5. Morariu C.O. (2014): Applied Statistics. Transilvania University of Braşov Publishing House, ISBN 978-606-190397-9, Brașov, Romania

6. Dobrițoiu N. (2018): Ingineria calităţii (Quality Engineering). Universitas Publishing House, Petroșani, Romania, ISBN 978-973-741-583-7 (in Romanian)

7. Clinciu R., Clinciu M.R. (2012): Estimating the Calibration life for Measuring Devices. Academic Journal of Manufacturing Engineering, ISSN 1583-7904, No. 10/2, p. 30-35

8. Clinciu M.R. (2009): Estimating The Statistical Parameters For Data Samples In An Experimental Installation. Proceedings of ModTech 2009, ISSN 2066-3919, p. 167-170, Iaşi, Romania 